\title{
Avaliação do equilíbrio de idosas praticantes de diferentes modalidades esportivas
}

\author{
Evaluation of the balance of elderly practicers of \\ different sports modalities
}

\section{Karen Rodrigues Euzébio', Jefferson Carlos Araujo Silva², Tamara Augusta Ferreira de Paiva ${ }^{3}$, Fernando Barcellar Biângulo ${ }^{4}$, Liana Mayara Queiroz Caland ${ }^{5}$, Lídia Mara de Aguiar Bezerra ${ }^{6}$}

\author{
${ }^{1}$ Centro Universitário Euro Americano. Brasília, Distrito Federal, Brasil. ORCID: 0000-0003-4599-8970.h2karen.edu.fisica@gmail.com \\ ${ }^{2}$ Autor para correspondência. Universidade de Brasília. Brasília, Distrito Federal, Brasil. ORCID: 0000-0001-9131-4360. jeffcasilva@gmail.com \\ 3Universidade de Brasília. Brasília, Distrito Federal, Brasil. ORCID: 0000-0002-2941-0199. tamisdp@gmail.com \\ ${ }^{4}$ Universidade de Brasília. Brasília, Distrito Federal, Brasil. ORCID: 0000-0001-8329-4224. fernandobarcellar@gmail.com \\ 5Universidade de Brasília. Brasília, Distrito Federal, Brasil. ORCID: 0000-0001-7752-9357. ft.liana@gmail.com \\ 6Universidade de Brasília. Brasília, Distrito Federal, Brasil. ORCID: 0000-0003-0661-6298. lidia.bezerra@gmail.com
}

RESUMO | INTRODUÇão: O processo natural de envelhecimento leva os sistemas do corpo humano ao declínio de suas funções. É consenso na literatura que a atividade física direcionada para idosos promove efeitos positivos na manutenção da saúde destes, promovendo redução da perda de massa muscular, ganho de flexibilidade e aumento da resistência cardiorrespiratória. OBJETIVO: Avaliar o equilíbrio de idosas praticantes de diferentes modalidades esportivas. MÉTODOS: A amostra de idosas integrantes de diferentes grupos de atividades esportivas, e idosas residentes em uma instituição de longa permanência. Todos os grupos possuíam 15 voluntárias, a saber: capoterapia (GCa), hidroginástica (GH), ginástica (GG), treinamento de força (GTF) e grupo controle (GC). As participantes tiveram sua massa corporal, estatura, cirtormetria da cintura e quadril avaliados. A avaliação do equilíbrio das voluntárias foi realizada através do teste de equilíbrio unipodal com e sem restrição visual, nesta ordem. As diferenças entre os grupos foram verificadas através da análise de variância ANOVA One Way, $p<0,05$. RESULTADOS: As atividades de treinamento de força surtiram efeito positivo no equilíbrio sem restrição visual, enquanto que as voluntárias da capoterapia demonstraram melhor desempenho no equilíbrio com restrição visual. CONCLUSÃO: Atividades que englobem diversas modalidades parecem ser mais eficientes para manter um adequado equilíbrio de idosos.
ABSTRACT | INTRODUCTION: The natural process of aging leads the systems of the human body to decline in function. There is a consensus in the literature that physical activity directed at the elderly promotes positive effects on maintaining their health, promoting reduction of muscle mass loss, flexibility gain and increased cardiorespiratory resistance. OBJECTIVE: To evaluate the balance of elderly women practicing different sports. METHODS: A sample of elderly women from different groups of sports activities, and elderly residents in a long-term institution. All groups had 15 volunteers, namely: capotherapy (GCa), water aerobics (GWA), gymnastics (GG), strength training (GST) and control group (GC). The volunteers had their body mass, height, waist cirtormetry and hip evaluated. The balance evaluation of the volunteers was performed through the unipodal balance test with and without visual restriction, in this order. Differences between groups were verified by one way ANOVA, $p<0,05$. RESULTS: The strength training activities had a positive effect on balance without visual restriction, while capotherapy volunteers showed better performance on balance with visual restriction. CONCLUSION: Activities that encompass several modalities seem to be more efficient to maintain an adequate balance of the elderly.

KEYWORDS: Elderly. Balance. Physical activity.

PALAVRAS-CHAVE: Idoso. Equilíbrio. Atividade física. 


\section{Introdução}

O processo natural de envelhecimento, típico aos seres humanos, caracteriza-se por seu caráter progressivo e gradual que conduz à perda das aptidões funcionais do organismo, com alterações psicológicas e motoras' ${ }^{1}$. No Brasil e no mundo tem-se observando um aumento dos indivíduos idosos, pelo Censo de 2010 o Brasil possuía um total de 20.590 .599 idosos, o que correspondia a $10 \%$ da população total. Atualmente, estima-se que a população de idosos seja de 26 milhões ${ }^{2}$.

O processo natural de envelhecimento leva os sistemas do corpo humano ao declínio de suas funções. O sistema musculoesquelético apresenta redução da força e massa muscular (sarcopenia), enquanto o sistema cardiovascular cursa com diminuição da capacidade de contração e adaptação a diferentes graus de esforço, o sistema nervoso, por sua vez, sofre com a atrofia e redução do volume do córtex, redução do número de neurônios e neurotransmissores. Este último apresenta íntima relação com a diminuição das respostas adaptativas de equilíbrio ${ }^{3,4}$.

Tais transformações advindas com o envelhecimento influenciam diretamente a qualidade de vida do idoso, em razão da limitação que os impõe, desta forma colocando sua saúde em maior vulnerabilidade. A redução da capacidade funcional dos sistemas implica diretamente na independência do idoso, tornando-o mais suscetível aos eventos adversos, tais como surgimento das Doenças Crônicas Não Transmissíveis (DCNT) ${ }^{5}$. A combinação dos déficits do organismo durante o processo natural de envelhecimento tem consequências diretas sobre o equilíbrio dos indivíduos idosos, na maioria das vezes proporcionando efeitos danosos ${ }^{6}$.

Dente as consequências que surgem com os déficits de equilíbrio, o evento queda merece destaque. A queda decorre de um evento acidental onde o sujeito muda de posição, indo para um nível mais baixo ao que se encontra ${ }^{7}$. A queda pode representar incapacidade, perda da função e levar à síndrome do imobilismo, além disso, ocasiona prejuízos físicos e psicológicos, provocados pela diminuição da autonomia e independência. A queda pode ser responsável por fraturas, como as de quadril, e por um elevado número de hospitalização em idosos ${ }^{8}$.
É consenso na literatura que a atividade física direcionada para idosos promove efeitos positivos na manutenção da saúde destes, promovendo redução da perda de massa muscular, ganho de flexibilidade e aumento da resistência cardiorrespiratória 9. A atividade física pode melhorar e/ou manter a capacidade de funcional de idosos, influenciando positivamente no controle e/ ou prevenção das DCNT associadas ao sedentarismo, como coronariopatias, Diabetes Mellitus (DM), hipercolesterolemia, Hipertensão Arterial Sistêmica (HAS); além de melhorar o equilíbrio e coordenação ${ }^{10}$.

Os exercícios físicos cooperam para postergar e minimizar os efeitos que o processo natural de envelhecimento repercute na população idosa. Desta forma a prática regular de atividade física é um aliado para reduzir a suscetibilidade ao evento queda em idosos, através do incremento que promove no equilíbrio destes indivíduos ${ }^{11,12}$, porém, os estudos não especificam qual tipo de atividade física é o mais adequado para reduzir os déficits de equilíbrio em idosos. Tendo em vista o exposto o presente artigo teve por objetivo avaliar o equilíbrio de idosas praticantes de diferentes modalidades esportivas.

\section{Método}

Trata-se de um estudo transversal, quantitativo, descritivo e observacional, realizado com idosas integrantes de diferentes grupos de atividades esportivas, e idosas residentes de uma instituição de longa permanência. Foram adotados como critérios de inclusão indivíduos idosos ( $\geq 60$ anos), praticantes da modalidade esportiva de maneira regular, com autonomia física e funcional, com liberação médica para realizarem determinada modalidade e que não fizessem uso de medicações que interferissem no equilíbrio postural. E como critérios de exclusão indivíduos portadores de déficit cognitivo e motor, ou doenças que influenciassem diretamente o equilíbrio dos mesmos.

As idosas praticantes de atividades físicas foram recrutadas em dois centros (Sesc Ceilândia e Vila Olímpica Parque da Vaquejada) que oferecem atividades para os idosos, dentre as quais foram inclusos no estudo, capoterapia (GCa, $n=15)$, hidroginástica $(G H, n=15)$, ginástica $(G G, n=15)$ e 
treinamento de força (GTF, $n=15)$. E os idosos que compuseram o grupo controle $(G C, n=15)$ foram recrutados na instituição São Vicente de Paula. Ambos os centros localizam-se no Distrito Federal.

A pesquisa se norteou pela resolução 466/2012 do Conselho Nacional de Saúde (CNS) que regulamenta os estudos com seres humanos, todos os procedimentos relacionados à coleta e análise dos dados se deram após aprovação do Comitê de Ética em Pesquisa da Universidade Católica de Brasília, sob o protocolo nº 014/2007. As voluntárias confirmavam sua participação no estudo por meio da assinatura do Termo de Consentimento Livre e Esclarecido (TCLE).

A coleta de dados dividiu-se em duas etapas, a primeira consistia numa entrevista onde eram coletados dados referentes a caracterização antropométrica e a cirtometria da cintura e quadril, esta para cálculo da Relação Cintura Quadril (RCQ). A classificação da RCQ divide os sujeitos em baixo risco (RCQ <0,76), moderado (RCQ entre 0,76 a 0,84 ) e alto risco (RCQ entre 0,85 a 0,90) 13. A segunda etapa se dava pela avaliação do equilíbrio das voluntárias através do teste de equilíbrio unipodal com e sem restrição visual, nesta ordem ${ }^{14}$.

Os dados foram apresentados na forma de estatística descritiva, utilizando-se os procedimentos de média e desvios-padrão. E no intuito de observar diferenças entre grupos uma análise de variância ANOVA One Way foi calculada com nível de significância adotado de $p<0,05$. Todos os dados foram calculados utilizando o programa SPSS versão 17.0.

\section{Resultados}

Os dados referentes a média de idade, massa corporal, estatura, Índice de Massa Corporal (IMC), cirtometria e RCQ de todos os cinco grupos encontram-se descritos na tabela $\mathrm{n}^{\circ} 01$.

Tabela 1. Médias de idade, massa corporal, estatura e cirtometria e classificações de idosos de dois centros do Distrito Federal, 2010

\begin{tabular}{|c|c|c|c|c|c|}
\hline Variáveis & $\begin{array}{c}\text { GC } \\
(n=15) \\
X \pm D P\end{array}$ & $\begin{array}{c}\text { GG } \\
(n=15) \\
X \pm D P\end{array}$ & $\begin{array}{c}\text { GH } \\
(n=15) \\
X \pm D P\end{array}$ & $\begin{array}{c}\text { GTF } \\
(n=15) \\
X \pm D P\end{array}$ & $\begin{array}{l}\text { GCa } \\
\begin{aligned} &(n=15) \\
& \pm D P\end{aligned}\end{array}$ \\
\hline Idade (anos) & $70.93 \pm 5.6^{\star}$ & $66.0 \pm 7.0$ & $67.4 \pm 4.7$ & $65.1 \pm 4.8$ & $62.9 \pm 2.8$ * \\
\hline Massa Corporal (kg) & $60.6 \pm 10.2$ & $71.6 \pm 13.2$ & $60.0 \pm 9.8$ & $64.9 \pm 8.3$ & $72.1 \pm 10.9$ \\
\hline Estatura (metros) & $1.48 \pm 0.05 t$ & $1.55 \pm 0.5$ & $1.50 \pm 0.5$ & $1.56 \pm 0.03 \dagger$ & $1.54 \pm 0.6$ \\
\hline $\mathrm{IMC}\left(\mathrm{kg} / \mathrm{m}^{2}\right)$ & $27.3 \pm 4.8$ & $30.2 \pm 6.0$ & $26.5 \pm 3.8$ & $26.5 \pm 3.4$ & $30.1 \pm 4.0$ \\
\hline Class.IMC & $1.7 \pm 0.8$ & $2.4 \pm 1.0$ & $1.6 \pm 0.7$ & $1.69 \pm 0.63$ & $2.3 \pm 0.8$ \\
\hline Cintura & $90.1 \pm 8.2$ & $92.4 \pm 9.3$ & $86.0 \pm 7.4$ & $86.3 \pm 6.7$ & $91.9 \pm 7.4$ \\
\hline Quadril & $100 \pm 8.8$ & $107.2 \pm 11.2$ & $99.2 \pm 9.4$ & $99.7 \pm 8.3$ & $104.2 \pm 5.7$ \\
\hline RCQ & $0.91 \pm 0.08$ & $0.85 \pm 0.05$ & $0.87 \pm 0.05$ & $0.86 \pm 0.05$ & $0.87 \pm 0.04$ \\
\hline Class. RCQ & Alto & Alto & Alto & Alto & Alto \\
\hline
\end{tabular}

IMC - Índice de Massa Corporal

RCQ - relação cintura quadril e Class.RCQ - classificação do risco cardíaco pela relação cintura quadril

*1 - Diferença significante grupo controle X grupo capoterapia

$\dagger^{2}$ - Diferença significante grupo controle $X$ grupo treinamento resistido (TR) 
Após a análise de variância (One Way) foi observada que na variável equilíbrio sem restrição visual, o GC alcançou valor inferior quando comparado com o GTF. Também foi mostrado valor inferior da mesma variável no GCa quando comparado com o GTF. Já para a variável equilíbrio com restrição visual o GC teve um tempo inferior quando comparado com o GTF e o mesmo também demonstrou inferioridade significante quando comparado com o GCa.

Tabela 2. Avaliação do equilíbrio de idosas praticantes de diferentes modalidades esportivas através do teste unipodal, Distrito Federal, 2010

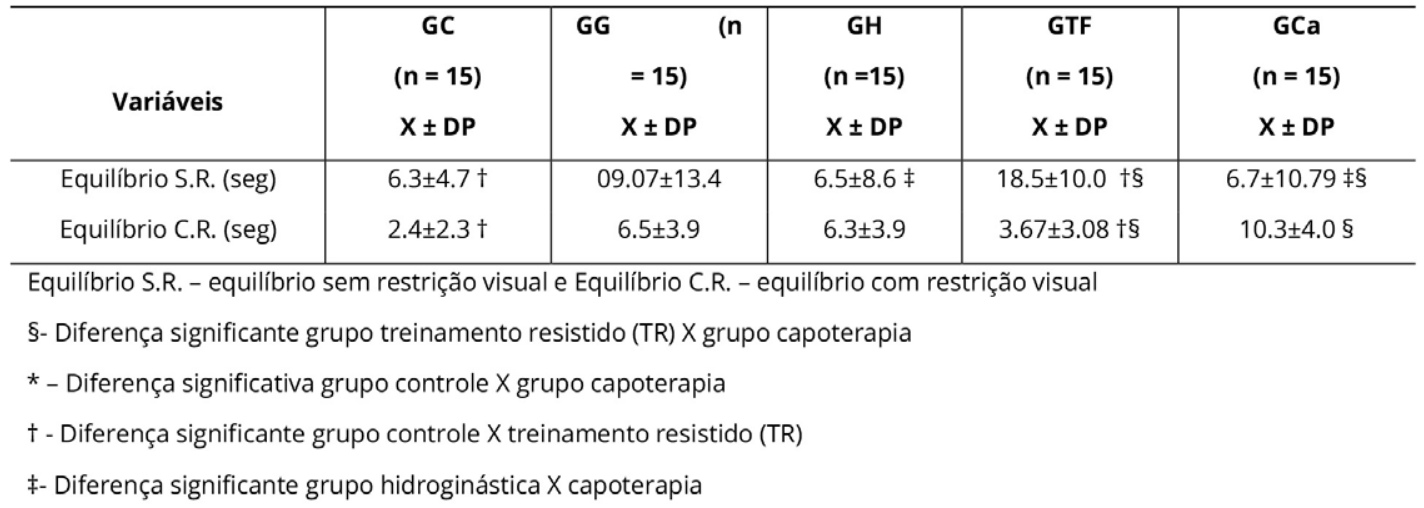

\section{Discussão}

O presente artigo teve por objetivo avaliar o equilíbrio, com e sem restrição visual, de idosas praticantes de diferentes modalidades esportivas. As alterações que decorrem do processo natural de envelhecimento influenciam diretamente o equilíbrio destes indivíduos, um conjunto de modificações morfológicas, funcionais, bioquímicas e psicológicas estão envolvidas na diminuição do desempenho funcional de idosos 5 . Este fato influencia o equilíbrio postural dos idosos, onde as alterações estruturais aliadas as perdas funcionais dos sistemas sensoriais e motor são responsáveis por ocasionar déficits no sistema mantenedor do equilíbrio ${ }^{15}$.

No presente estudo a média de idade do GC foi superior à dos demais grupos, apresentando, ainda, menor estatura e maior classificação de risco cardíaco pela RCQ. Os voluntários do GC não praticavam atividades físicas e eram residentes de uma instituição de longa permanência. Um estudo ${ }^{16}$ realizado em São Caetano do Sul-SP avaliou a associação entre a marcha e a capacidade funcional e aptidão física em idosos institucionalizados, 69 voluntários de ambos os sexos foram avaliados através dos testes de velocidade normal de andar, velocidade máxima de andar, cadência de passos, teste unipodal de 30 segundos e Timed Up and Go (TUG). Os autores evidenciaram que quanto maior a idade menor o comprimento da passada e do passo, por outro lado quanto maior o tempo de equilíbrio estático, maior a amplitude da marcha. No presente estudo o GC apresentou menores médias de equilíbrio nas duas modalidades, com e sem restrição visual, sugere-se que a não realização de atividade física, de maneira regular, possa ter contribuído para tais achados.

A avaliação da RCQ demonstrou que todos os voluntários dos grupos apresentavam valores considerados de alto risco. Uma pesquisa ${ }^{17}$ realizada em MG avaliou a RCQ de 8 idosas participantes de um grupo de ginástica, este possuía uma frequência semanal de 2 vezes, com duração de 60 minutos, as voluntárias tiveram sua massa corporal e estatura mensuradas para o cálculo do IMC, e a cirtometria da cintura e quadril para o cálculo da RCQ. Os autores indentificaram três voluntárias com moderada RCQ, quatro com alta RCQ e uma idosa com RCQ muito alta. Apesar de em ambos os estudos os voluntários serem praticantes de atividade física, exceto o grupo controle do presente estudo, a presença de RCQ alta chama atenção para os riscos de eventos cardiovasculares a que estão expostos os idosos, e os incentivos as práticas de atividade física de maneira regular devem ser constantes, para minimizar tais efeitos ${ }^{18}$. 
Na avaliação do equilíbrio sem restrição visual o GG apresentou médias superiores ao GC, GH e GCa, no entanto, não superou o GTF. Na avaliação do equilíbrio com restrição visual o GG apresentou médias superiores o GC, GH e GTF, porém, o a média do GCa foi superior nesse quesito. A ginástica é uma atividade que melhora a flexibilidade dos indivíduos e proporciona uma manutenção da Amplitude de Movimento (ADM), agilidade, equilíbrio e coordenação, importante nas articulações dos MMII para realização da marcha ${ }^{19}$.

Um ensaio clínico ${ }^{20}$ teve por objetivo avaliar se os efeitos do treinamento de equilíbrio e coordenação motora, sistematizado e supervisionado, reduziria a propensão a quedas em idosos. Todos voluntários já realizavam protocolo de reabilitação cardiopulmonar e aos indivíduos do grupo experimental foram acrescidos o treinamento de equilíbrio e coordenação motora, os exercícios foram realizados três vezes por semana, durante 12 semanas. Os autores puderam concluir que o protocolo de equilíbrio e coordenação motora surtiu efeitos positivos na melhora do equilíbrio estático e dinâmico e na coordenação, diminuindo a propensão a quedas dos voluntários que participaram do grupo experimental. No presente estudo, todos os participantes, exceto os do GC, apresentaram desempenho satisfatório nos testes de avaliação do equilíbrio, isso reflete o efeito protetor que a prática regular de atividade física promove, minimizando os déficits de equilíbrio que o processo natural de envelhecimento ocasiona, desta forma tornando os idosos menos susceptíveis ao evento queda ${ }^{15,21}$.

A população idosa apresenta redução da prática regular de atividade física, isso influencia diretamente no surgimento de DCNT e na redução da capacidade funcional, sua prática deve estimulada, pois associa-se diretamente a melhora na qualidade de vida e independência para realização de suas atividades ${ }^{22}$. O GH apresentou menor IMC que os grupos GC, GG e GCa, porém apresentou resultado muito parecido ao do GTF, na classificação do IMC, o GH demonstrou classificação inferior aos demais grupos, fato que deve ter sido influenciado pela estatura média do grupo.
Um estudo ${ }^{23}$ realizou a comparação de três protocolos de exercícios, exercícios no solo, exercícios na água e grupo controle, 132 voluntários tiveram sua flexibilidade, força abdominal e dos MMII e equilíbrio avaliados antes e ao final de oito semanas de treinamento. Os resultados mostraram que ao final do protocolo os voluntários apresentaram melhora da força muscular, do equilíbrio e da flexibilidade, no entanto, para o grupo que realizou exercícios na água, foram observadas melhoras nas medidas de força muscular abdominal e equilíbrio. Nossos resultados mostraram que o $\mathrm{GH}$ apresentou resultados superiores apenas ao GC, quando avaliado o equilíbrio sem restrição visual, na avaliação do equilíbrio com restrição visual o $\mathrm{GH}$ demonstrou médias a frente do GTF e GC. Estes resultados podem sofrer influência em razão dos princípios físicos da água a que estão expostos os praticantes de hidroterapia ${ }^{24}$.

Idosos apresentam redução da massa muscular, sarcopenia, de forma gradual, sendo observados alterações estruturais e remodelamento das unidades motoras $^{5}$. O treinamento resistido direcionado aos idosos pode retardar essa perda de massa muscular, influenciando no equilíbrio corporal e reduzindo os riscos de quedas, proporcionando maior independência9. Quando avaliado o equilíbrio sem restrição visual no presente estudo, o GTF apresentou melhores resultados em comparação aos demais, na avaliação com restrição visual o GCa apresentou média superior. Uma pesquisa ${ }^{21}$ desenvolvida com idosas de Porto Alegre-RS comparou dois tipos de treinamento, força muscular e flexibilidade, e sua influência no equilíbrio, ambos os protocolos de treinamento possuíram 11 semanas de duração com frequência de duas vezes por semana, para avaliar o equilíbrio utilizou-se a Escala de Equilíbrio de Berg (EEB). Os autores observaram que ambos os grupos apresentaram aumento significativo no equilíbrio corporal após aplicação do protocolo, implicando para uma independência funcional efetiva e menor risco de quedas. 
A capoterapia é uma atividade baseada na capoeira voltada para o público idoso, engloba exercícios de alongamento e fortalecimento muscular, promovendo benefícios como melhora da coordenação motora, função cognitiva e socialização ${ }^{25}$. Sugere-se que em razão desse fato as voluntárias do GCa apresentaram melhores resultados na avaliação do equilíbrio com restrição visual. Valendo-se de uma integração de movimentos coordenados em associação com a música, a capoterapia, além de uma prática que utiliza movimentos da capoeira, estimula a brincadeira, o lúdico, a diversão, o bem-estar corporal, entre outros benefícios. Estes são pontos importantes a serem integrados nas atividades voltadas para os idosos, pois o processo de envelhecimento é associado ao quadro depressivo, que contribui para isolamento social e diminuição da independência funcional ${ }^{26}$.

O estudo apresentou como limitações a não investigação do tempo de prática de cada atividade. Mais pesquisas são necessárias avaliando a combinação de modalidades e observando o tempo de frequência de cada tipo de treinamento para gerar repercussões positivas no equilíbrio corporal de idosos e minimizar as chances de queda nesses indivíduos.

\section{Conclusão}

A avaliação do equilíbrio de idosas que praticam diferentes modalidades esportivas com e sem restrição visual, evidenciou que as atividades voltadas para o treinamento de força possuíram melhores respostas no equilíbrio sem restrição visual, enquanto que as voluntárias que realizavam atividades de capoterapia apresentaram melhor equilíbrio frente a avaliação com restrição visual. Todas as voluntárias do estudo apresentaram RCQ considerada alta, evidenciando a propensão a riscos cardiovasculares a que estão expostos, apesar de praticarem atividade física, exceto o GC.

Uma combinação de atividades que envolvam as mais variadas modalidades esportivas parece ser o mais adequado para minimizar os déficits que 0 processo natural de envelhecimento ocasiona no equilíbrio corporal dos indivíduos.

\section{Contribuição dos autores}

Euzébio KR participou da concepção, delineamento, coleta dos dados, busca e análise estatística dos dados da pesquisa, interpretação dos resultados, redação do artigo científico. Silva JCA, Paiva TAF e Biângulo FB participaram da redação do artigo científico. Caland LMQ participou da revisão crítica do artigo. Bezerra LMA participou da concepção, delineamento, busca e análise estatística dos dados da pesquisa, bem como da interpretação dos resultados.

\section{Conflitos de interesses}

Nenhum conflito financeiro, legal ou político envolvendo terceiros (governo, empresas e fundações privadas, etc.) foi declarado para nenhum aspecto do trabalho submetido (incluindo mas não limitando-se a subvenções e financiamentos, participação em conselho consultivo, desenho de estudo, preparação de manuscrito, análise estatística, etc.).

\section{Referências}

1. Ministério da Saúde. Envelhecimento e saúde da pessoa idosa. Série A. Normas e Manuais Técnicos. Caderno de Atenção Básica. Brasília: Ministério da Saúde; 2006.

2. DATASUS. Indicadores demográficos. Proporção de idosos na população. [Internet]. [acesso em 22 de dez de 2017]. Disponível em: http://tabnet.datasus.gov.br/cgi/tabcgi.exe?idb2011/a14.def

3. Martins JAO, Miranda DG, Malaman TAB, Leite SN. Efeitos de um programa de exercícios de fortalecimento muscular na prevenção de quedas em idosos da comunidade. Revista Inspirar: Movimento \& Saúde. 2014; 6(1):18-21.

4. Marin L, Lima S, Giacomin L. Avaliação da qualidade de vida de idosos com e sem correção de valvulopatia cardíaca: relatos de casos. FisiSenectus, 2014; 2(1): 43-50. doi: 10.22298/rfs.2014. v2.n1.2896

5. Fong JH, Feng J. Comparing the loss of functional independence of olders adults in the U.S. and China. Archives of Gerontology and Geriatrics, 2018; 74: 123-127. doi: 10.1016/j. archger.2017.10.020

6. Almeida ST, Soldera CLC, Carli GA, Gomes I, Resende TL. Análise de fatores extrínsecos e intrínsecos que predispõem a quedas em idosos. Rev Assoc Med Bras, 2012; 58(4):427-433. doi: 10.1590/ S0104-42302012000400012 
7. Prata HL, Junior EDA, Paula FL, Ferreira SM. Envelhecimento, depressão e quedas: um estudo com os participantes do Projeto Prev-Quedas. Fisioter Mov, 2011;24(3):437-43. doi: 10.1590/S0103$\underline{51502011000300008}$

8. Alves RLT, Silva CFM, Pimentel LN, Souza ACS, Coelho LAF, Costa IA. Avaliação dos fatores de risco que contribuem para queda em idosos. Rev Bras Geriatr Gerontol, 2017; 20(1): 59-69. doi: 10.1590/1981-22562017020.160022

9. Gillespie LD, Robertson MC, Gillespie WJ, Lamb SE, Gates S, Cumming RG et al. Interventions for preventing falls in older people living in the community. Cochrane Database of Systematic Reviews 2009; (9):CD007146. doi: 10.1002/14651858.CD007146. pub3

10. Kendrick D, Kumar A, Carpenter H, Zijlstra GAR, Skelton DA, Cook JR et al. Exercise for reducing fear of falling in older people living in the community. Cochrane Database of Systematic Reviews 2014; (11):CD009848. doi: 10.1002/14651858.CD009848. pub2

11. Silva PCR, Oliveira VH, Neto ECA, Azevedo KPM, Rebouças GM, Knackfuss MI. Impacto do agachamento em superfície estável e instável sobre o equilíbrio estático e dinâmico de idosos. Rev Andal Med Deporte, 2017; 10(4):176-180. doi: 10.1016/j. ramd.2015.09.004

12. Carvalho DA, Brito AF, Santos MAP, Nogueira FRS, Sá GGM, Neto JO et al. Prevalência da prática de exercícios físicos em idosos e sua relação com as dificuldades e a falta de aconselhamento profissional específico. R. bras. Ci. e Mov. 2017;25(1):29-40. doi: 10.18511/rbcm.v25i1.6467

13. Lohman TG, Roche AF, Martorell R. Anthropometrics and standardization reference manual. Illinois: Human Kinetics Book; 1988.

14. Alfiere FM, Riberto M, Gatz LS, Ribeiro CPC, Battistella LR. Uso de testes clínicos para verificação do controle postural em idosos submetidos a programas de exercícios físicos. Acta Fisiatr, 2010; 17(4): 153-158.

15. Woellner SS, Araujo AGS, Martins JS. Protocolos de equilíbrio e quedas em idosos. Neurociências, 2014;10(2): 104-117.

16. Beltran DCG, Junior JPS, Mancini RB, Araújo TL, Matsudo SMM. Relação do padrão de marcha associado com a aptidão física e a capacidade funcional de residentes de instituições de longa permanência. Estud. Intediscipl. Envelhec, 2017; 22(2): 43-55.
17. Soares PG, Pádua TV. Relação entre cintura-quadril e imagem corporal em mulheres de meia idade e idosas ativas fisicamente. Revista Kairós Gerontologia, 2014;17(1): 283-295.

18. Pereira MWM, Arruda AL, Lima MS, Martins KMS, Damacena $K G$, Alves GSA et al. Indicadores antropométricos associados a fatores de risco cardiovasculares em idosos. Rev Eletrônica Gestão \& Saúde. Saúde do Idoso, 2014;5(5): 3115-31.

19. Ribeiro DP, Mazo GZ, Brust C, Cardoso AS, Silva AH, Benedetti TRB. Programa de ginástica para idosos nos centros de saúde: avaliação da aptidão funcional. Fisioterapia e Movimento, 2009; 22(3): 407-417.

20. Ferreira JP, Cruz VL, Cardoso RA, Leite FNTS, Duarte CMG, Gouveia VM. Efeitos do treinamento de equilíbrio e coordenação motora em idosos: ensaio clínico randomizado. Geriatria \& Gerontologia, 2012; 6(2): 183-191.

21. Albino ILR, Freitas CR, Teixeira AR, Gonçalves AK, Santos AMPV, Bós AJG. Influência do treinamento de força muscular e de flexibilidade articular sobre o equilíbrio corporal em idosas. Rev Bras Geriatr Gerontol, 2012;15(1):17-25. doi: 10.1590/S1809$\underline{98232012000100003}$

22. Vagetti GC, Oliveira V, Silva MP, Pacífico AB, Costa TRA, Campos W. Associação do índice de massa corporal com aptidão funcional de idosos participantes de um programa de atividade física. Rev Bras Geriatr Gerontol, 2017;20(2):216-227. doi: 10.1590/1981-22562017020.160160

23. Padua E, Campoli F, Manzi V, Panzarino M, Lombardo M, Melchiorri $G$ et al. Water versus land-based exercises as physical training programs in elderly. The jornal os sports medicine and physical fitness, 2018; 58(6): 802-9. doi: 10.23736/500224707.17.07307-8

24. Vieira JR, Alves MO, Luzes R. Efeitos da hidroterapia em pacientes idosos com osteoartrose de joelho. Rev discente da UNIABEU, 2016; 4(8): 11-15.

25. Freire GV, Silva IP, Moura WB, Rocha FCV, Madeira MZA, Amorim FCM. Perfil de idosos que frequentam um centro de convivência da terceira idade. R Interd, 2015; 8(2): 11-19.

26. Prazeres MMV, Mendes MT, Sousa IRC, Mazzocante RP, Maciel DG, Ribeiro C et al. Capoterapia como lazer e atividades física lúdica para idosos: uma percepção dos capoterapeutas. Licere, 2016; 18(4): 320-340. 